\title{
Efeito do óleo essencial de Tagetes patula L. (Asteraceae) sobre Sitophilus zeamais Motschulsky (Coleoptera, Curculionidae)
}

\author{
Rozane Maria Restello ${ }^{1}$, Cristiane Menegatt ${ }^{2} \&$ Altemir José Mossi ${ }^{1}$
}

${ }^{1}$ Programa de Pós-Graduação em Ecologia, Departamento de Ciências Biológicas, Universidade Regional Integrada do Alto Uruguai e das Missões, Av. Sete de Setembro 1621, 99700-000 Erechim-RS, Brasil. rrozane@uricer.edu.br

${ }^{2}$ Programa de Pós-Graduação em Ecologia, URI-Campus Erechim - RS, crismenegatt@yahoo.com.br

\begin{abstract}
Effect of the essential oil of Tagetes patula L. (Asteraceae) on Sitophilus zeamais Motschulsky (Coleoptera, Curculionidae). Countless problems due to the use of chemical insecticides for the control of pests in stored grains have been observed. In order to minimize these problems, alternative measures have been developed, including the use of botanical insecticides. This work was carried out with the objective of evaluating the effect of the essential oil of Tagetes patula L. on, Sitophilus zeamais (Mots., 1855) in corn kernels in laboratory conditions. The T. patula essential oil was obtained through hidrodestilation with the Clevenger device. The chemical characterization of the compounds found in the essential oil of $T$. patula was carried by gas chromatography connected to the mass spectrometer. The compounds found were: limonene $(37.05 \%)$, terpinolene $(32.61 \%)$, piperitone $(14.40 \%)$, neophitadiene $(5.91 \%)$, sabinene $(2.88 \%)$, trans-ocimene $(2.02 \%)$, beta-cariphilene $(1.98 \%)$, farnesol $(1.84 \%)$, and alpha-pinene $(1.30 \%)$. The insects used in the bio-test were kept under controled temperature and relative humidity environment. Two bioassays were carried out to evaluate the effect of the essential oil of $T$. patula on the behavior (activity/repellence) and insecticide activity on adults of $S$. zeamais. The results showed that this essential oil was efficient (concentration of $10 \mu \mathrm{L}$ ) to control adults of $S$. zeamais adults.
\end{abstract}

KEYWORDS. Control; repellence; bioactyve composites; beetle of maize.

RESUMO. Efeito do óleo essencial de Tagetes patula L. (Asteraceae) sobre Sitophilus zeamais Motschulsky (Coleoptera, Curculionidae). Resistência de pragas a inseticidas, danos ao ambiente em função da poluição do solo e de recursos hídricos vem sendo observados pelo uso de inseticidas químicos no controle de pragas em grãos armazenados. Com o intuito de minimizar estes problemas, estão sendo estudadas alternativas como o uso de inseticidas botânicos. Este trabalho foi realizado com o objetivo de avaliar o efeito do óleo essencial de Tagetes patula L. sobre, Sitophilus zeamais Motschulsky, 1855 em milho sob condições de laboratório. O óleo essencial de T. patula foi obtido através de arraste a vapor com aparelho clevenger. A caracterização química dos compostos encontrados no óleo essencial de T. patula foi realizada através de cromatografia gasosa acoplado a espectrometria de massas, sendo observados: limoneno (37,05\%), terpinoleno $(32,61 \%)$, piperitone $(14,40 \%)$, eofitadieno $(5,91 \%)$, sabineno $(2,88 \%)$, trans-ocimeno $(2,02 \%)$, beta-cariofileno $(1,98 \%)$, farnesol $(1,84 \%)$ e alfa-pineno $(1,30 \%)$. Os insetos utilizados nos bioensaios foram mantidos em temperatura e umidade relativa controlada. Foram avaliados os efeitos do óleo essencial de T. patula sobre o comportamento (atratividade e/ou repelência) e atividade inseticida sobre adultos de $S$. zeamais através de dois bioensaios. Pelos resultados pode-se observar o efeito repelente e inseticida do óleo essencial de T. patula sobre S. zeamais, na concentração de 10 $\mu \mathrm{L}(\mathrm{p}<0,0001$ e $\mathrm{p}=0,02)$ sendo portanto, eficaz no controle de adultos de $S$. zeamais.

PALAVRAS-CHAVE. Controle; repelência; compostos bioativos; gorgulho-do-milho.

A produção brasileira de grãos na safra de 2006/2007 foi estimada em 130,5 milhões de toneladas, destacando-se entre outras, as culturas de soja, milho, arroz e trigo, sendo estas de grande importância no cenário agrícola do Brasil (Companhia Nacional de Abastecimento - Conab 2008). Mundialmente, a produção de milho chega a 615,92 milhões de toneladas, atrás apenas do trigo (Cocamar 2004).

Os insetos causam freqüentemente danos extensivos aos grãos armazenados e aos produtos destes grãos. As perdas quantitativas podem atingir $5-10 \%$ nas regiões temperadas e 20-30\% nas regiões tropicais (Haque et al. 2000). De acordo com Silva et al. (2007) no Brasil, as perdas devido ao ataque de pragas chegam à $20 \%$ da produção total do grão, demandando a necessidade de novas pesquisas e tecnologias.

O inseto com maior importância em grãos armazenados é o gorgulho-do-milho Sitophilus zeamais Motschulsky, 1855. Este inseto apresenta elevado potencial biótico, grande número de hospedeiros, infestação cruzada e facilidade na penetração na massa de grãos (Gallo et al. 2002).

$\mathrm{O}$ uso indiscriminado de agentes químicos para combater insetos encontrados em grãos armazenados causa diversos danos. Desta forma, torna-se relevante a busca de novos compostos para uso no manejo e controle de pragas, sem problemas como: a contaminação ambiental, resíduos nos alimentos, efeitos prejudiciais sobre os organismos benéficos e surgimento de insetos resistentes, entre outros. Este aspecto tem despertado o interesse de vários pesquisadores com relação ao uso de extratos vegetais para este fim.

Um grande número de substâncias derivadas de plantas possui atividades fisiológicas e comportamentais sobre insetos de produtos armazenados (Ho et al. 1996; Rajedran \& Sriranjini 2008). Essas substâncias naturais são freqüentemente oriundas 
do metabolismo secundário das plantas e podem ser utilizadas de diferentes formas. Segundo Prates \& Santos (2000) dentre os metabólitos secundários encontram-se os terpenos, especialmente os monoterpenos e seus análogos, sendo estes componentes abundantes em óleos essenciais de plantas superiores. Estes podem reduzir a produtividade de insetos de produtos armazenados, afetando o crescimento, desenvolvimento e reprodução de alguns insetos herbívoros. Os pesticidas naturais baseados em óleos essenciais de plantas podem representar uma alternativa de proteção aos produtos armazenados (Isman 2000).

O uso de substâncias de origem vegetal no controle de pragas de grãos armazenados é muito promissor. Procópio et al. (2003) avaliando o fator de repelência de pós de seis espécies vegetais sobre adultos de Sitophilus zeamais, verificou que a planta que provocou maior repelência aos mesmos foi Eucalyptus citriodora (Hook) (Myrtaceae). Já em relação à atividade inseticida, a planta que apresentou eficiência foi Chenopodium ambrosioides L. (Chenopodiaceae), que provocou a mortalidade dos insetos.

Uma das plantas utilizadas tanto para controle de insetos, como para atividade antimicrobiana bem como para controle de fungos e nematicidas tem sido o Tagetes patula (Boueri \& Lunardi (2006). Esta planta caracteriza-se por ser de fácil cultivo, bastante decorativa e de ciclo relativamente longo. A utilização de produtos naturais tem apresentado resultados satisfatórios, pois além da fácil aquisição, preparo, utilização, possuem baixo custo de produção, favorecendo principalmente o pequeno agricultor (Silva et al. 2007). Considerando estas boas perspectivas, este trabalho foi realizado com o objetivo de avaliar o efeito do óleo essencial de Tagetes patula L. (Asteraceae), conhecida como cravo-de-defunto, sobre Sitophilus zeamais em milho armazenado sob condições de laboratório.

\section{MATERIALE MÉTODOS}

Extração do óleo essencial de Tagetes patula. O óleo essencial utilizado nos experimentos foi obtido das folhas e caules de Tagetes patula, cultivadas na unidade experimental da Universidade Regional Integrada do Alto Uruguai e Missões - URI - Campus de Erechim.

Após a floração de Tagetes patula, as plantas foram coletadas e secas em estufa a temperatura de $30^{\circ} \mathrm{C}$ na ausência de luz e com aeração. $O$ processo utilizado para a extração do óleo essencial foi a hidrodestilação. Para cada extração foram utilizados $100 \mathrm{~g}$ de folhas e caules secos imersos em um balão de $5 \mathrm{~L}$ com $3 \mathrm{~L}$ de água destilada acoplado em um extrator clevenger.

Caracterização química. Para a identificação dos compostos do óleo essencial, utilizou-se cromatografia gasosa acoplada à espectrometria de massa, pois este é um dos métodos mais indicados para identificação de compostos de óleos essências (Castro et al. 2004; Simões \& Spitzer 2001). O aparelho utilizado foi CG-EM (Shimadzu, Modelo QP 5050A), com coluna capilar DB-5 (30 m X 0,25 mm X 0,25 $\mu$ m de espessura do filme), vazão do gás de arraste (hélio) de $1,0 \mathrm{~mL}$.min- 1 , detector de $1.2 \mathrm{Kv}$. Temperatura do injetor $280^{\circ} \mathrm{C}$ e interface a $300^{\circ} \mathrm{C}$ e tempo de corte do solvente em $4 \mathrm{~min}$. As amostras foram preparadas na concentração de 1000 ppm de óleo com 300 ppm de padrão interno (bifenila) em $1 \mathrm{~mL}$ utilizando-se diclorometano (Merck) como solvente, sendo injetado um volume padrão de $1 \mu \mathrm{L} \mathrm{em}$ triplicata. Os picos foram integrados no modo manual e a identificação dos compostos foi feita por comparações do espectro de massas com os espectros existentes nos banco de dados do equipamento (Wiley). Os compostos majoritários foram listados e semiquantificados.

Bioensaios com Sitophilus zeamais e óleo essencial de Tagetes patula. Os insetos utilizados nos bioensaios foram mantidos no Laboratório de Biotecnologia da URI - Campus de Erechim, sob condições adequadas de temperatura $\left(25 \pm 2^{\circ} \mathrm{C}\right)$ e umidade relativa $(65 \pm 10 \%)$, em vidros de boca larga com capacidade para $1 \mathrm{~L}$ vedados com um tecido fino, contendo grãos de milho previamente tratados, como substrato de alimentação, utilizados em todos os bioensaios.

Os insetos foram separados ao acaso e mantidos sem alimento por três horas antes do preparo dos bioensaios, conforme sugerido por Prates \& Santos (2000). Para os bioensaios foi utilizado o óleo essencial puro obtido anteriormente.

Avaliação da repelência. Para avaliação da repelência de Tagetes patula sobre Sitophilus zeamais, foi utilizada uma arena formada por cinco caixas plásticas circulares $(6,0 \mathrm{~cm}$ de diâmetro e 2,0 cm de altura), sendo a caixa central interligada simetricamente às demais por tubos plásticos, dispostos diagonalmente (Mazzonetto \& Vendramim 2003; Procópio et al. 2003; Tavares \& Vendramim 2005).

Amostras de milho (30 g), impregnadas uma hora antes da montagem do experimento, com óleo essencial $(10 \mu \mathrm{L})$ de Tagetes patula foram colocadas em duas caixas plásticas. Nas duas caixas simetricamente opostas foram colocadas apenas amostras de milho ( $30 \mathrm{~g}$ ) sem óleo essencial (testemunha). No recipiente central foram liberados 50 adultos, não sexados, e após $24 \mathrm{~h}$ contou-se o número de insetos por recipiente.

A partir dos dados observados no teste, foi aplicado o Índice de Preferência (I.P.), citado por Procópio et al. (2003) onde:

$$
\text { I.P. }=\frac{(\% I P T-\% I p t)}{(\% I P T+\% I p t)}
$$

I.P.= Índice de Preferência

$\% \mathrm{IPT}=\%$ de insetos na planta-teste

$\% \mathrm{Ipt}=\%$ de insetos na testemunha

onde que: I.P.: - 1,00 a - 0,10, planta teste repelente; $-0,10$ a $+0,10$, planta teste neutra $\mathrm{e}+0,10 \mathrm{a}+1,00$, planta teste atraente.

Avaliação da atividade inseticida. Para este bioensaio foram utilizadas placas de Petri com o fundo forrado por papel filtro impregnado com a concentração de óleo a ser testada. Sobre esta, foi colocada uma camada de pérolas de vidro e acima mais uma camada de papel filtro, evitando assim o contato direto dos insetos com o óleo essencial. Foram realizadas cinco repetições para cada concentração de óleo testada $(5 \mu \mathrm{L}, 10$ 
$\mu \mathrm{L}, 20 \mu \mathrm{L}, 30 \mu \mathrm{L}, 50 \mu \mathrm{L})$ e a testemunha $(0 \mu \mathrm{L})$, sendo liberados 20 insetos adultos em cada placa. As placas foram observadas após 24 horas de inoculação do óleo, contando-se o número de insetos mortos por placa.

Para analisar a eficácia das concentrações do óleo essencial de T. patula sobre S. zeamais utilizou-se a fórmula de Abbot (1925) modificada.

$$
\text { Eficácia }(\%)=\frac{\text { mortalidade tratamento }- \text { mortalidade testemunha }}{100-\text { mortalidade testemunha }}
$$

Análise estatística dos dados. Para avaliar a diferença entre a repelência do óleo essêncial frente à testemunha, utilizou-se a análise de variância (ANOVA one way) com o Teste Tukey a posteriori. O efeito da atividade inseticida do óleo essencial foi avaliado a partir do cálculo da regressão linear simples considerando a concentração do óleo como variável preditora e a porcentagem de eficácia como variável resposta.

\section{RESULTADOS EDISCUSSÃO}

Caracterização Química. Na caracterização química do óleo essencial de Tagetes patula destacam-se os picos de número 3 (limoneno) e o de número 6 (terpinoleno) que apresentam, em média, as maiores áreas nas amostras, 37,05\% e 32,61\%, respectivamente. $\mathrm{O}$ terceiro composto com maior área foi piperitone com $14,40 \%$. Os demais compostos selecionados neste estudo apresentam áreas com valores inferiores a 6\%: neofitadieno $(5,91 \%)$, sabineno $(2,88 \%)$, trans-ocimeno $(2,02 \%)$, beta-carifileno (1,98\%), farnesol $(1,84 \%)$ e alfa-pineno $(1,30)$ (Tabela I, Fig. 1).

Cestari et al. (2004) citaram que plantas de T. minuta são ricas em compostos secundários incluindo monoterpenos, sesquiterpenos, flavonóides e tiofenes. Sagar et al. (2005) estudando a composição química do óleo essencial de Tagetes patula no norte da Índia, comentam que limoneno, terpinoleno e piperitone foram os compostos majoritários, vindo a corroborar com os dados aqui apresentados.

Bioensaios com Sitophilus zeamais e óleo essencial de Tagetes patula

Avaliação da repelência. Conforme observado na Tabela II foi constatada atividade repelente em todos os bioensaios realizados, $4 \%$ dos insetos utilizados nos mesmos preferiram a caixa com milho tratado ( $10 \mu \mathrm{L}$ de óleo essencial de Tagetes patula).

Os resultados obtidos para o Índice de Preferência (Tabela II) demonstram que Tagetes patula é repelente para adultos de Sitophilus zeamais na concentração de $10 \mu \mathrm{L}$ de óleo essencial. Pela análise de variância foi verificado diferença significativa entre a testemunha e este tratamento (F 1,8 = $7689,9 ; \mathrm{p}<0,0001)$.

Rajendran \& Sriranjini (2008) estudando o efeito fumegante de óleos essenciais de várias plantas, comentam do efeito tóxico sobre ovos e pupas de Tribolium castaneum, Rhyzoperta dominica, Sitophilus oryzae e Sitophilus zeamais corroborando com os dados aqui apresentados.

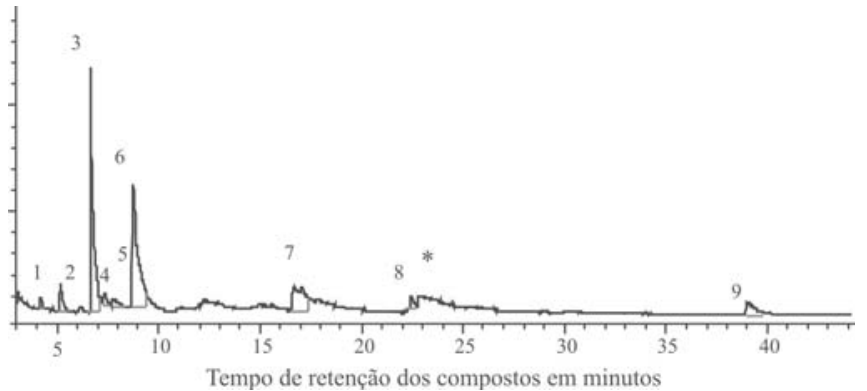

Fig. 1. Cromatograma do óleo essencial de Tagetes patula obtido através de cromatografia gasosa acoplada a espectometria de massas. 1, alfa pineno; 2, sabineno; 3, limoneno; 4, trans ocimeno; 5, farnesol; 6, terpinoleno; 7, piperitone; 8, beta-cariofileno; 9, neofitadieno.

Avaliação da atividade inseticida. Nos bioensaios realizados neste estudo, observou-se a atividade inseticida (fumigação) do óleo essencial de Tagetes patula sobre adultos de Sitophilus zeamais, em condições de laboratório. Pode-se perceber que com maiores concentrações do óleo essencial 30 e $50 \mu \mathrm{L}$ a mortalidade dos insetos foi de $100 \%$. Com $20 \mu \mathrm{L}$ a eficácia foi de $97 \%$, seguida de $94 \%(10 \mu \mathrm{L})$ e $81 \%(5 \mu \mathrm{L})$. Nas placas sem tratamento (testemunha) não houve mortalidade de insetos. No entanto, verificou-se que o óleo essencial de Tagetes patula tem efeito significativo em todos os tratamentos, mas fica mais evidente nas concentrações inferiores a $10 \mu \mathrm{L}(\mathrm{F}$ $1,4=12,7 ; \mathrm{p}=0,02)$. De acordo com Prates \& Santos (2000) observou-se o efeito knock down, isto é, efeito de choque caracterizado pela incapacidade de caminhar e com evolução para a morte.

Resultado semelhante encontrou Nascimento et al. (2008), onde 96 a 100\% de adultos de Sitophilus zeamais morreram quando o extrato de Tagetes patula foi aplicado na forma de vapor sobre os adultos.

Segundo Prates \& Santos (2000) os inseticidas mais eficientes apresentam ação por contato e/ou ingestão e ação fumigante, e, nesse caso a pressão de vapor (taxa de evaporação) é um fator importante. Os monoterpenos cineol e limoneno possuem grande ação inseticida em relação a importantes pragas de grãos armazenados. Essas substâncias

Tabela I. Lista de compostos selecionados dos cromatogramas após análise por cromatografia gasosa acoplada a espectrometria de massa de flores de Tagetes patula.

\begin{tabular}{cccc}
\hline Picos & Tempo de retenção & Área $(\%)$ & Composto \\
\hline 1 & 4,175 & 1,30 & Alfa pineno \\
2 & 5,200 & 2,88 & Sabineno \\
3 & 6,650 & 37,05 & Limoneno \\
4 & 7,300 & 2,02 & Trans ocimeno \\
5 & 7,767 & 1,84 & Farnesol \\
6 & 8,733 & 32,61 & Terpinoleno \\
7 & 17,108 & 14,40 & Piperitone \\
8 & 22,475 & 1,98 & Beta-cariofileno \\
9 & 39,100 & 5,91 & Neofitadieno \\
\hline
\end{tabular}

*Após o pico de número oito há um pico significativo que não foi citado aqui, por não ser um composto do óleo essencial da planta. Este pico é o padrão interno utilizado na amostra (bifenila). 
Tabela II. Atratividade de adultos de Sitophilus zeamais pelo óleo essencial de Tagetes patula e Índice de Preferência (I.P.) calculado.

\begin{tabular}{cccccc}
\hline $\begin{array}{c}\text { Teste de } \\
\text { repelência }\end{array}$ & $\begin{array}{c}\text { Testemunha } \\
\text { (sem óleo) }\end{array}$ & $\begin{array}{c}\text { Tratamento } \\
(10 \text { ì })\end{array}$ & $\%$ & I.P. \\
\hline 1 & 48 & $96 \%$ & 2 & $4 \%$ & $-0,92$ \\
2 & 49 & $98 \%$ & 1 & $2 \%$ & $-0,96$ \\
3 & 48 & $96 \%$ & 2 & $4 \%$ & $-0,92$ \\
4 & 47 & $94 \%$ & 3 & $6 \%$ & $-0,87$ \\
5 & 49 & $98 \%$ & 1 & $2 \%$ & $-0,96$ \\
\hline
\end{tabular}

* Na caixa central do bioensaio foram liberados 50 insetos, sem o substrato de alimentação dos mesmos (milho).

são tóxicas através da penetração no corpo do inseto via sistema respiratório (efeito fumigante), através da cutícula (efeito de contato) e pelo aparelho digestivo (efeito de ingestão).

Segundo Keita et al. (2000), espécies do gênero Tagetes têm apresentado atividade inseticida contra pragas de produtos armazenados. Perich et al. (1995) apud Cestari et al. (2004) citaram que terpenos do óleo essencial de Tagetes minuta L. são responsáveis pelo efeito tóxico apresentado em mosquito.

Estudos com outras plantas, como o caso do trabalho de Liu \& Ho (1999), com óleo essencial de Evodia rutaecarpa Juss. (Rutaceae) observaram que este óleo mostrou forte efeito repelente sobre Tribolium castaneum Herbst. (Tenebrionidae) e efeito repelente moderado sobre Sitophilus zeamais. Pós de frutos e da planta inteira (com frutos) de Chenopodium ambrosioides apresentam efeito altamente tóxico ao gorgulhodo-milho, além de reduzirem a emergência dos adultos (Tavares \& Vendramim 2005). Em estudos realizados com óleo de Allium sativum (L.) (Liliaceae), Ho et al. (1996) relataram a mortalidade e redução na emergência de adultos de Tribolium castaneum e Sitophilus zeamais.

Diante do exposto, o óleo essencial de Tagetes patula tem efeito repelente e inseticida sobre Sitophilus zeamais, porém existem ainda muitas questões a serem analisadas para esclarecer vantagens, desvantagens e/ou limitações no uso de substâncias de origem botânica no controle de insetospraga, inclusive para outras plantas nativas da região Alto Uruguai do Rio Grande do Sul, sob as características climáticas encontradas.

Agradecimentos. Agradecemos a Márcio Aurélio Garcia Correia Tavares e ao professor Doutor José Djair Vendramim pelas importantes sugestões durante a realização do trabalho.

\section{REFERÊNCIAS}

Abbot, W. S. 1925. A method for computing the effectivness of an insecticide. Journal of Economic Entomology 18: 265-267.

Bouri, M. A. \& D. M. C. Lunardi. 2006. Avaliação de elementos agrometeorológicos no cultivo de cravo-de-defunto (Tagetes sp.) em ambiente protegido e a campo. Revista de Energia na Agricultura 21: 45-54.
Castro, H. G.; L. O. Oliveira; L. C. A. Barbosa; F. A. Ferreira; D. J. H. Silva; P. R. Mosquim \& E. A. Nascimento. 2004. Teor e composição do óleo essencial de cinco acessos de Mentrasto. Química Nova 27: $55-57$.

Cestari, I. M.; S. J. Sarti; C. M. Waib \& Jr. A. Branco. 2004. Evaluation of the potential insecticide activity of Tagetes minuta (Asteraceae) essential oil against the head lice Pediculus humanus capitis (Phthiraptera: Pediculidae). Neotropical Entomology 33: 805807.

COCAMAR - Cooperativa Agroindustrial de Maringá. 2004. Informativo: Produção de milho. Disponível em: http:// www.cocamar.com.br. Acesso em: 10 de outubro de 2008.

Companhia Nacional de Abastecimento. Disponível em: http:// www.conab.gov.br. Acesso em: 13 de outubro de 2008.

Gallo, D.; O. Nakano; S. Silveira Neto; R. P. L. Carvalho; G. C. Baptista; E. Berti Filho; J. R. P. Parra; R. A. Zucchi; S. B. Alves; J. D. Vendramim; L. C. Marchini; J. R. S. Lopes \& C. Omoto. 2002. Manual de Entomologia Agrícola. Piracicaba, SP: FEALQ, 920p.

Haque, M. A.; H. Nakakita; H. Ikenaga \& N. Sota. 2000. Developmentinhibiting activity of some tropical plants against Sitophilus zeamais Motschulsky (Coleoptera: Curculionidae). Journal of Stored Products Research 36: 281-287.

Ho, S. H.; L. Koh; Y. Ma; Y. Huang \& K. Y. Sim.1996. The oil of garlic, Allium sativum L. (Amaryllidaceae), as a potential grain protectant against Tribolium castaneum (Herbst) and Sitophilus zeamais Motsch. Journal of Stored Products Research 9: 41-48.

Isman, M. B. 2000. Plant essential oils for pest and disease management. Crop Protection 19: 603-608.

Keita, S. M.; C. Vicent; J. Schmit; S. Ramaswamy \& A. Belanger. 2000. Effect of various essential oils on Callosobruchus maculates (F.) (Coleoptera:Bruchidae). Journal of Stored Products Research 36: $355-364$.

Liu, Z. L. \& S. H. Ho. 1999. Bioactivity of the essencial oil extracted from Evodia rutaecarpa Hook f. et Thomas against the grain storage insects, Sitophilus zeamais Motsch. and Tribolium castaneum (Herbst). Journal of Stored Products Research 35: $317-328$.

Mazzonetto, F. \& J. D.Vendramim. 2003. Efeito de pós de origem vegetal sobre Acanthoscelides obtectus (Say) (Coleoptera: Bruchidae) em feijão armazenado. Neotropical Entomology 32: $145-149$.

Nascimento, F. J.; E. T. Diniz Filho; L. X. Mesquita; A. M. de Oliveira \& T. F. C. Pereira. 2008. Extractos Vegetales en el Control De Plagas. Revista Verde 3: 01-05.

Prates, H. T. \& J. P. Santos. P. 2000. Óleos essenciais no controle de pragas de grãos armazenados, p. 443-461. In: Lorini, I.; L. H. Miike; V. M. Scussel. Armazenagem de Grãos. Campinas: IBG, $1000 \mathrm{p}$.

Procópio, S. O.; J. D. Vendramim; J. I. Ribeiro Júnior \& J. B. Santos. 2003. Bioatividade de diversos pós de origem vegetal em relação à Sitophilus zeamais Mots. (Coleoptera: Curculionidae). Ciência e Agrotecnologia 27: 1231-1236.

Rajedran, S. \& V. Sriranjini. 2008. Plants products as fumigants for stored-product insect control. Journal of Stored Products Research 44: 126-135.

Sagar, D. V.; S. N. Naik; P. K. Rout \& Y. R. Rao. 2005. Composition of essencial oils of Tagetes patula L. growing in Northern India. Journal of Essencial Oil Research 17: 446-448.

Silva, P. H.; P. C. O. Trivelin; N. Guirado; E. J. Ambrosano; P. C. D. Mendes; F. Rossi \& R. A. Arévolo. 2007. Controle alternativo de Sitophilus zeamais MOTS., 1855 (Col.: Curculionidae) em grãos de milho. Revista Brasileira de Agroecologia 2: 902-905.

Simões, C. M. O. \& V. Spitzer. 2001. Óleos Voláteis, p. 397-425. In: Simões, C. M. O. (Org.). Farmacognosia: Da Planta ao Medicamento. $3^{\text {a }}$ ed. Porto Alegre/ Florianópolis: Ed. Universidade UFRGS/ Ed. da UFSC, 821 p.

Tavares, M. A. G. C. \& J. D. Vendramim. 2005. Bioatividade da Ervade-Santa-Maria, Chenopodium ambrosioides L., sobre Sitophilus zeamais Mots. (Coleoptera: Curculionidae). Neotropical Entomology 34: 319-323. 